\title{
Cannonball appearance, a tricky yet underrated imaging manifestation of COVID-19
}

\author{
Javid Azadbakht $^{1} \cdot$ Hamidreza Talari $^{1} \cdot$ Delbar Rastkhiz $^{1} \cdot$ Mahsa Masjedi Isfahani $^{1}$
}

Received: 7 September 2021 / Accepted: 4 November 2021 / Published online: 13 November 2021

(c) American Society of Emergency Radiology 2021

\begin{abstract}
Spreading swiftly across the borders and over the seas, severe acute respiratory syndrome-related coronavirus-2 (SARS-COV-2), as causative pathogen of coronavirus disease 2019 (COVID-19), is currently the main global public health concern. "Cannonball appearance," as a rare and yet underrated CT feature of COVID-19 pneumonia, has been typically linked to certain hematogenous pulmonary metastases and some inflammatory/infection conditions, including tuberculosis, but no other viral or atypical pneumonia. Cannonball appearance can bring diagnostic dilemmas and difficulties in monitoring treatment response in patients with or suspicious for hematogenous pulmonary metastasis. Hereby, we report two cases of COVID-19 delta variant-induced pneumonia manifesting unusually in chest CT scan with cannonball appearance.
\end{abstract}

Keywords COVID-19 $\cdot$ Delta variant $\cdot$ Cannonball appearance $\cdot$ Metastasis $\cdot$ Pneumonia $\cdot$ SARS-CoV-2

\section{Introduction}

Coronavirus disease 2019 (COVID-19) has been troubling healthcare systems all around the world since late Dec 2019, crossing borders devastatingly and at a fast pace, spread to over 200 different countries; as it has infected more than 214 million people, and claimed nearly 4.5 million lives as of August 25, 2021 [1]. In Jan 2020, COVID-19 was declared as a Public Health Emergency of International Concern by the World Health Organization [2]. The reference standard to diagnose COVID-19 disease is real-time reverse-transcription polymerase chain reaction (rRT-PCR), that not uncommonly returns a false-negative result, especially earlier in the disease course [3]. Alternatively, chest CT scan provides both diagnostic and prognostic value, with an accuracy even higher than that of rRT-PCR for detecting COVID-19 [4]. In COVID-19-induced pneumonia, chest CT scan mostly demonstrates bilateral, basilar, peripheral, wedge-shaped, and pleural base opacities of ground-glass opacity (GGO) or less likely consolidative attenuation, halo

Mahsa Masjedi Isfahani

mahsami141@gmail.com

1 Department of Radiology, Faculty of Medicine, Shahid Beheshti Hospital, Kashan University of Medical Sciences, Qotb Ravandi Blvd., 8715981151 Kashan, Iran sign (central denser focus surrounded by a halo of GGO), and reverse halo appearance (central region of GGO surrounded by a band of consolidation) [5-7]. Beside these common manifestations, COVID-19 pneumonia may present with a variety of less common CT features, one of least reported example of which is "cannonball appearance." To our knowledge, this CT finding has been previously reported in only one case [8]. Cannonball appearance refers to multiple large, well-demarcated, round, or oval pulmonary nodules, resembling cannonballs in shape. This CT feature is typically linked to pulmonary metastases, mainly from renal cell carcinoma and choriocarcinoma $[9,10]$, and less frequently from prostate, endometrial carcinoma, and adrenal carcinomas or synovial sarcoma [10-12]. Rarely, schwannoma metastasis to lungs and pulmonary diffuse large B-cell lymphoma may present as cannonball appearance [13]. Aside from malignant causes, some inflammatory/infectious causes could be the culprit as their granulomas mat present as cannonballs, including granulomatosis with polyangiitis, fungal infections (e.g., histoplasmosis, cryptococcosis, and nocardiosis), and tuberculosis [14].

Hereby, we present two cases of rRT-PCR confirmed COVID-19-induced pneumonia manifested with cannonball appearance on CT scan. This specific CT finding needs to be taken a comprehensive view of, as it can lead to diagnostic perplexities or monitoring dilemmas. 


\section{Case 1}

A 39-year-old woman presented with fever, chills, dry cough, and malaise; her past medical history of chronic mild depression (being on long-term oral chlordiazepoxide, $10 \mathrm{mg}$ bd); otherwise, history was unremarkable. On day 4 (4 days post-symptom onset), patient referred to emergency department and visited by an infectious disease specialist. Patient was conscious and vital signs were stable. Air-room SPO2 was measured by pulse oximeter as $96 \%$. On arrival, patient underwent chest CT scan and nasopharyngeal and oropharyngeal swab sampling for rRT-PCR result returned positive. Genomic sequencing revealed infection with SARS-CoV-2 delta variant. Nasopharyngeal secretion culture tested negative for common bacterial pathogens. First CT (Fig. 1a) showed some scattered multilobar peripheral consolidative nodule-like densities in all lung lobes bilaterally. Some of nodules showed halo appearance (central denser focus surrounded by a halo of GGO), and others were of GGO. Remdesivir )200 $\mathrm{mg}$ IV stat, and then $100 \mathrm{mg}$ daily for next 4 days: total dose of 600mg) and dexamethasone (8mg PO stat, then 4 $\mathrm{mg}$ daily for next 4 days) were prescribed and patient was discharged home with discharge instructions on self-isolation and home-care. On day 8, patient referred to emergency department complaining of continuing dry cough, tachypnea, fever, and chills. Axillary temperature on arrival was $38.4{ }^{\circ} \mathrm{C}$ and pulse oximetry showed an air-room SPO2 of 97\%; vital signs were stable. Chest CT was performed and showed a rise in number, size, and density of nodule-like lesions (Fig. 1b-d). Some of nodules showed halo appearance, and others demonstrated a reverse halo appearance (central region of GGO surrounded by a band of consolidation). Patient was discharged to home, while being informed to continue the self-isolation at home and to return in case of clinical deterioration.

On day $10+$, fever and chills subsided; however, patient's malaise and dry cough became more severe and she developed with ageusia and anosmia. On day $12+$, patients became febrile for second time (after 2-day period of being afebrile) and developed with hemoptysis. On day 13 in third chest CT scan, a slight increase in lesions' size and number were seen (Fig. 2). At the day of writing this manuscript, patient experienced an almost full recovery, with remaining mild malaise on the day 15 post-symptoms onset.
Fig. 1 Cannonball appearance in a 39-year-old female patient with COVID-19 delta variant pneumonia (case 1). First $\mathrm{CT}$ after 4 days (a) reveals two peripheral consolidative nodule-like densities (arrows) in right middle and lower lobes. One of the nodules in the right middle lobe shows halo appearance. In second CT after 8 days (b-d), nodules are larger in size (arrows) and one of them shows reverse halo appearance (curved arrow). Arrowheads demarcate skipped regions of pleural thickening, reactive to patchy subpleural inflammatory changes. Asterisk indicates a mass-like heterogenous consolidation with halo appearance

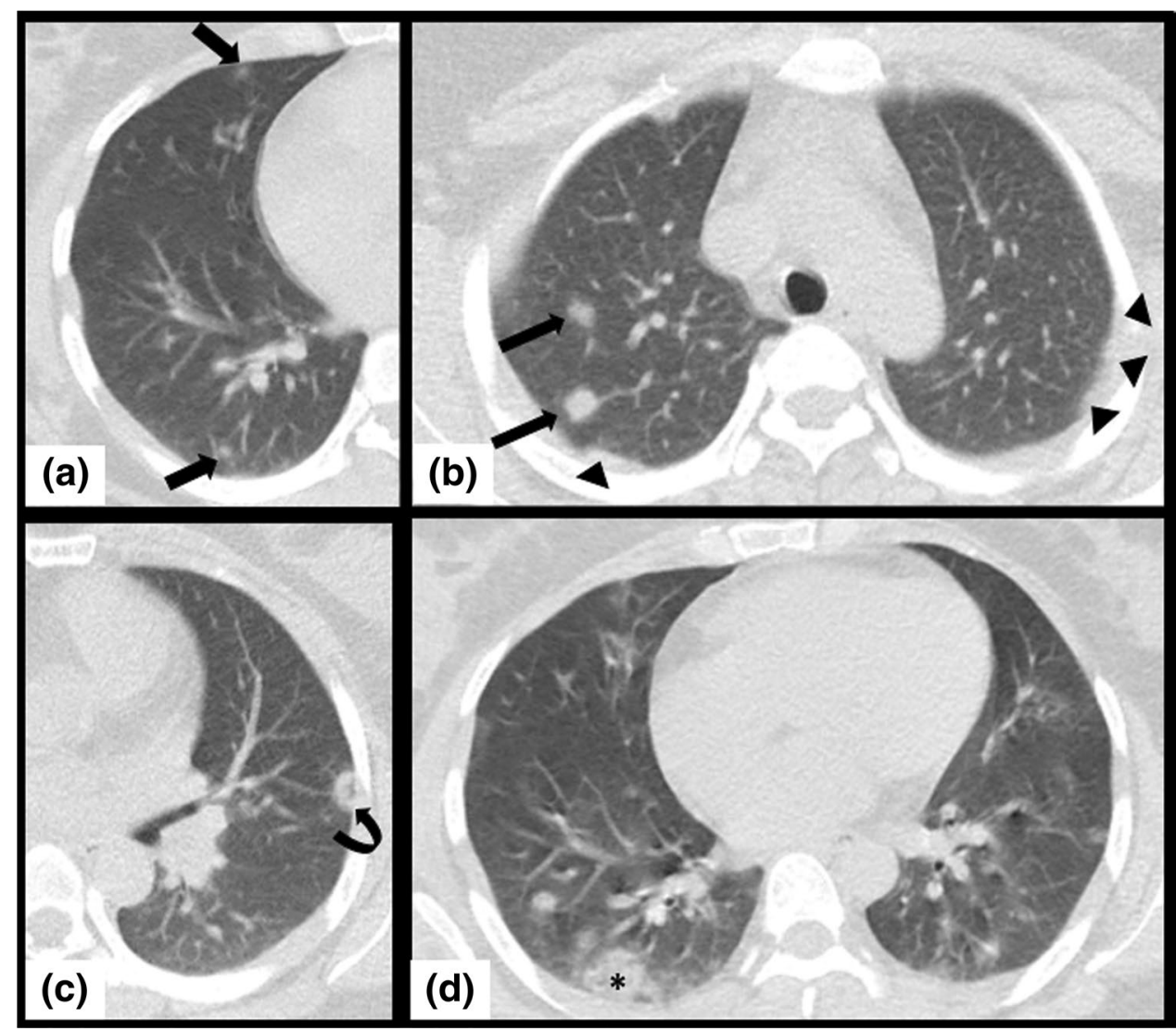




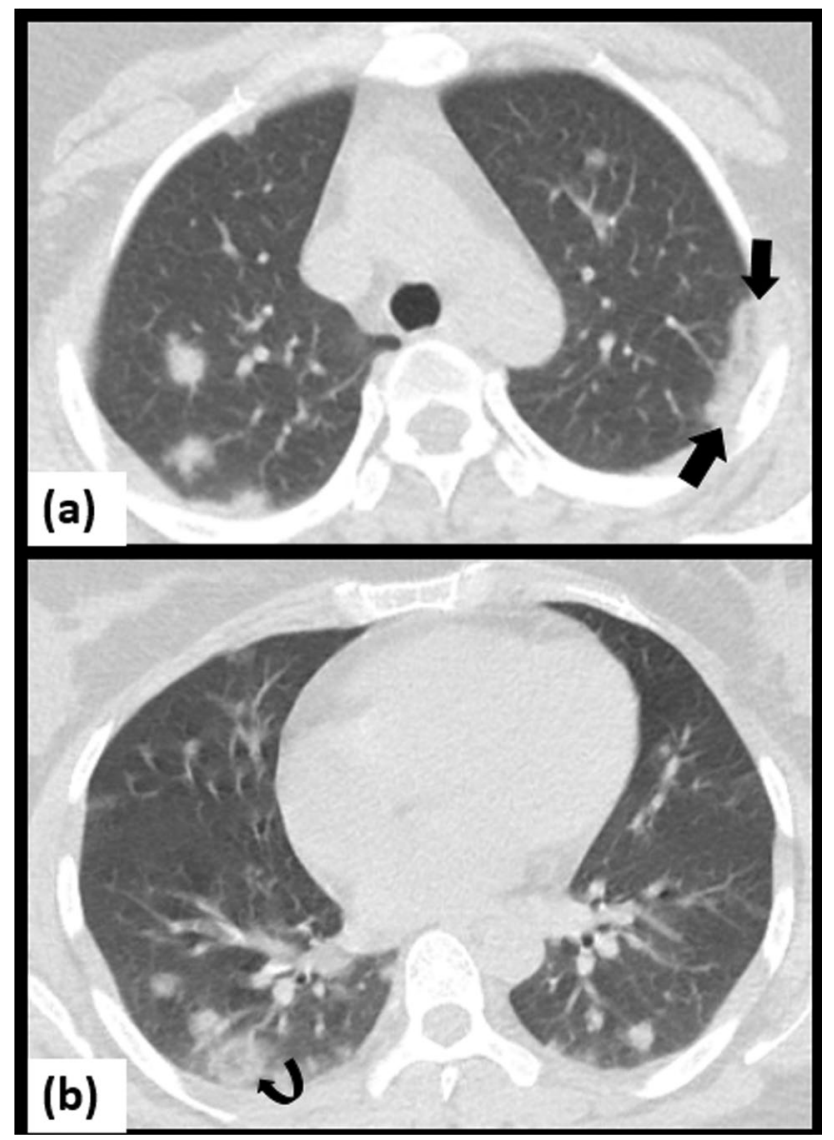

Fig. 2 a, b Third CT of the case 1, after 13 days, depicts subpleral consolidation (arrows) expanded over the pleural thickening which is shown in Fig. 1b. Mass-like consolidation, shown in d, is retaining a reverse halo appearance (asterisk)

\section{Case 2}

A 42-year-old diabetic male referring to emergency department with sore throat, dyspnea, and dry cough for 4 days. Patient had a history of previous intensive care unit (ICU) admission for diabetic ketoacidosis (DKA) a few months ago. On day 4+, patient was admitted to emergency department. Patient had a random blood sugar (BS) of $452 \mathrm{mg} / \mathrm{dl}$, blood pressure of $139 / 98 \mathrm{mmHg}$, pulse rate of 88 , and respiratory rate of 32. On physical examination, patient was ill and distressed, drowsy, tachypneic with difficult and abdominal breathing, and in auscultation had bilateral basilar crackles. Patient was mildly febrile (axillary temperature of $37.7^{\circ} \mathrm{C}$ ). Laboratory data showed a mild leukocytosis (white blood count of $12.3 \mathrm{k} / \mathrm{uL}$ ), and SPO2 with $\mathrm{O} 2$ therapy via nasal cannula was $96 \%$. Patient underwent chest CT scan, and remdesivir )200 mg IV stat, and then $100 \mathrm{mg}$ daily for next 4 days: total dose of $600 \mathrm{mg}$ ) and pantoprazole (40 mg IV qd) were started. RT-PCR testing on nasopharyngeal swab sample and genome sequencing revealed SARS-CoV-2 delta variant infection, while RT-PCR testing for influenza A and $\mathrm{B}$ viruses returned negative. CT showed multilobar consolidative nodule-like densities in all lung lobes bilaterally, with both central and peripheral distribution (Fig. 3).

On day 6+, patient's level of consciousness gradually decreased to Glasgow Coma Scale (GCS) of 11 while urine analysis, fasting BS, and venous and arterial blood gas (VBG and $\mathrm{ABG}$ ) showed evidence of diabetic ketoacidosis, and he was ICU admitted. On ICU admission, urine analysis demonstrated ketonuria $2+$ and glucosuria $3+$, and fasting BS was measured to be $426 \mathrm{mg} / \mathrm{dl}$. Blood culture was negative for common bacterial pathogens. In VBG, blood PH and bicarbonate level of 7.04 and $3.8 \mathrm{mmol} / \mathrm{L}$ were recorded
Fig. 3 a-c Mass-like consolidative lesions in a 42-year-old diabetic male patient with COVID-19 delta variant pneumonia (case 2). First CT after 4 days demonstrates multilobar scattered clusters of nodules in both lungs (arrows)

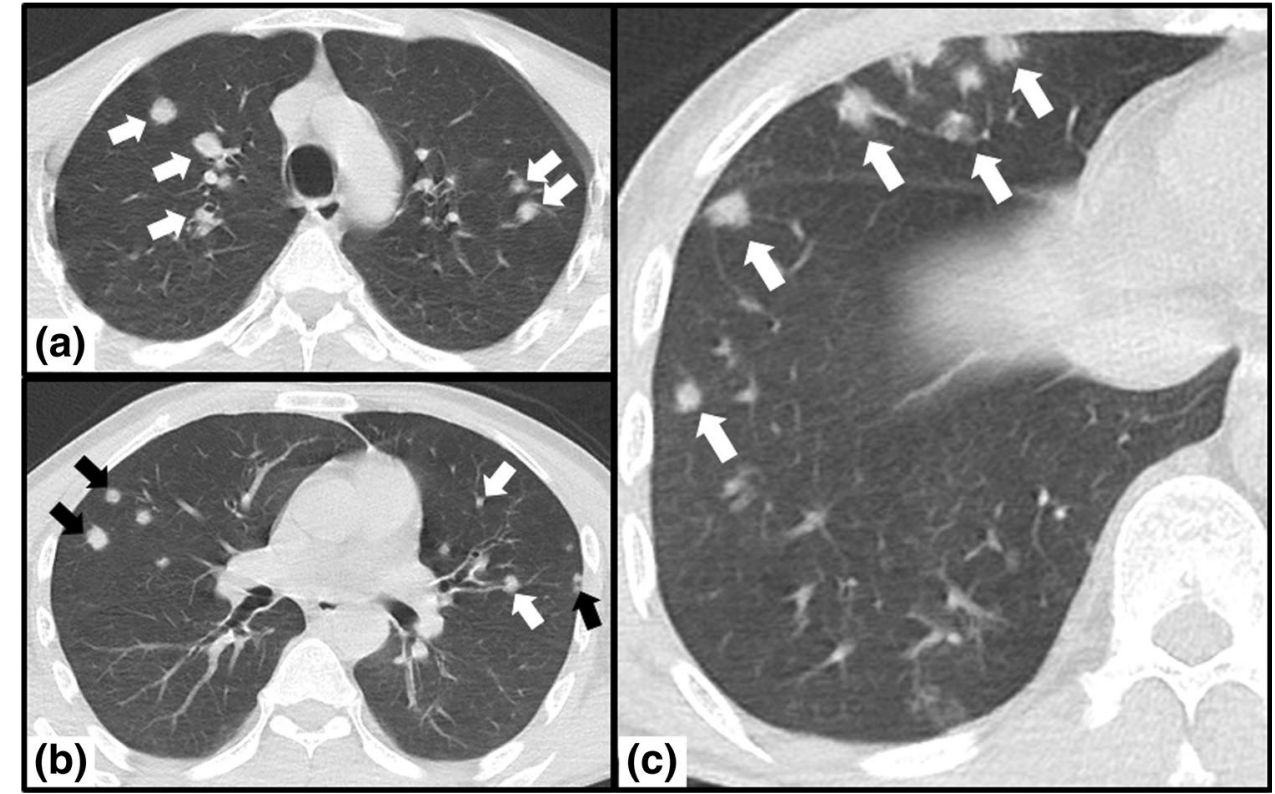


respectively. On $\mathrm{ABG}$, blood $\mathrm{PH}$ and lactate level were measured 7.19 and $2.2 \mathrm{mmol} / \mathrm{L}$. With the diagnosis of DKA, $300 \mathrm{cc}$ Half-Saline, $150 \mathrm{cc}$ bicarbonate $8.4 \%$, and regular insulin ( 7 units/h IV continuous infusion) were started and infused during $45 \mathrm{~min}$. After $2 \mathrm{~h}$, VBG results were as follows: blood PH, 7.27 and bicarbonate level $6.4 \mathrm{mmol} / \mathrm{L}$. On day 7 , WBC increased to $17.5 \mathrm{k} / \mathrm{uL}$ with marked neutrophilia (97.9\%) and lymphopenia (1.5\%); Imipenem (500 mg IV stat, and then $500 \mathrm{mg} \mathrm{bd}$ ) was ordered to empirically target any potential bacterial superinfection, and patient underwent chest CT scan for second time (Fig. 4). Comparing to case 1 , in case 2 , a drastic enlargement in the size and number of the lesions (forming heterogenous mass-like consolidations) was noticed. Lesions significantly changed in morphology (irregular margins with surrounding GGO which denote leading edge of inflammation, and central tiny foci of cavitation in some of lesions). Tracheal aspirate culture yielded no detectable fungal or bacterial infective agent, but tested positive for COVID-19 in RT-PCR. On day 8, VBG and urine analysis again became abnormal showing evidence of DKA (blood PH, 7.05; bicarbonate level, $3 \mathrm{mmol} / \mathrm{L}$; ketonuria, 4+; glucosuria, 4+; and fasting BS, $410 \mathrm{mg} / \mathrm{dL}$ ). Eventually patient deceased with cardiac arrest.

\section{Discussion}

Chest CT has been and is continuing to be the most widely implemented imaging modality to diagnose COVID-19 pneumonia and in some cases triage patients throughout the globe. Even low-dose chest CT scan is capable of diagnosing COVID-19 pneumonia accurately [15]. All common CT patterns of COVID-19 pneumonia have been reported and discussed thoroughly in literature. According to a meta-analysis, COVID-19 mainly presents with multilobar peripheral GGOs, although it may less commonly manifest as unilateral, consolidative, net-like, cord-like, nodular, or central lesion(s) [5]. Both cases reported in this study manifested unusually and atypically in chest CT scan, nodule-like or mass-like consolidative lesions being the most salient findings in imaging. Cannonball appearance in some studies has been linked to organizing pneumonia [16], and organizing pneumonia has been confirmed both radiographically and histologically in some of patients with COVID-19 pneumonia [17].

According to literature, it might be difficult to differentiate between COVID-19 pneumonia and other viral pneumonias, such as seasonal flu, merely relying on chest CT [18]. Cannonball appearance, although is not yet notorious for COVID-19, but has not been reported in atypical viral pneumonias; thus, it may help to differentiate COVID-19 pneumonia from other viral pneumonias and prevent delay in patient's isolation early on and before rRT-PCR confirms the diagnosis.

On the other hand, COVID-19-related pneumonia may obscure both morphological and metabolic features of metastatic cannonball lesions, bringing about diagnostic and monitoring dilemmas. For instance, in patients with metastatic renal cell carcinoma with cannonball appearance, the 18F-fluorodeoxyglucose (FDG) uptake by COVID-19-related lesions in the background may add spill-in counts to the total 18F-FDG uptake in metastatic lesions, resulting in a false high uptake in the metastatic deposits $[9,19]$.

Based on our experience, COVID-19 pneumonia manifesting with cannonball-like lesions in CT scan seems to be much more frequent in patients infected with SARS-CoV delta variant than in those infected with the older variants of the virus, although this statement is not currently supported by the literature and needs to be verified. Large-scale studies are warranted to validate, test, and explain this hypothesis.

In general, being familiar with cannonball appearance on chest CT imaging of patients with COVID-19 pneumonia can help to differentiate COVID-19 pneumonia from other atypical and viral pneumonias and prevent delay in patient's isolation, when confirmatory test results have not yet returned. Additionally, it emphasizing on a diagnostic
Fig. 4 a, b Second CT of the case 2 , after 7 days, reveals a remarkable enlargement in the size and number of lesions (arrows). Some of the clusters of nodules became confluent to form heterogenous mass-like opacities with irregular borders (arrowheads). Curved arrow indicates a small central cavitation in one of the lesions in right middle lobe

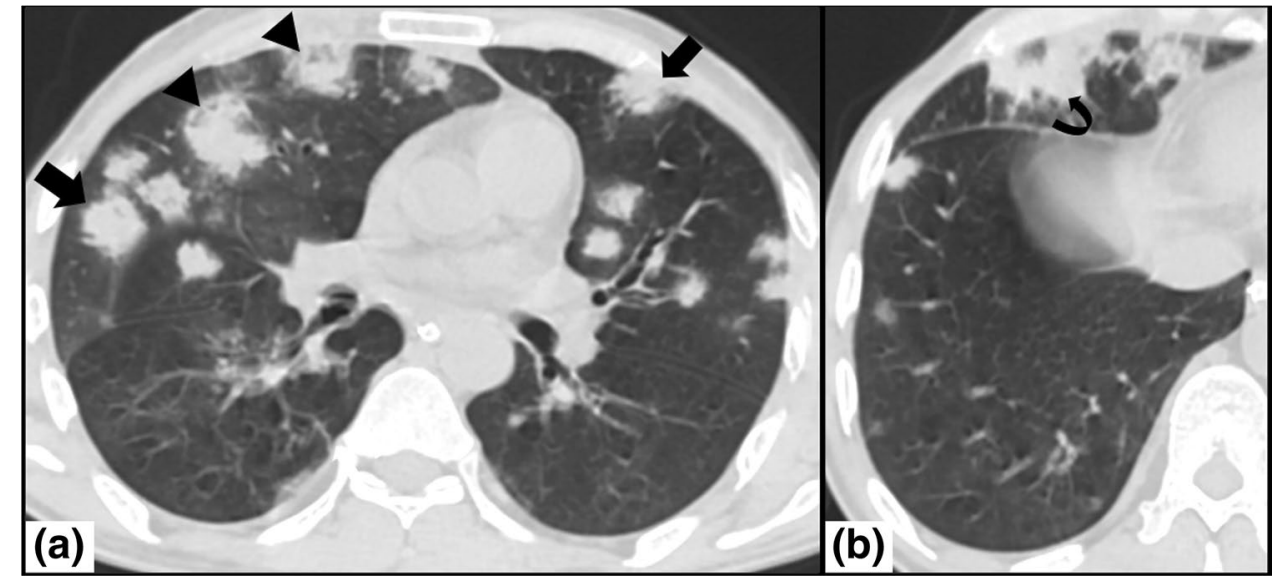


challenge: In patients with primary malignancy with possibility of cannonball metastases, in this time of COVID-19 pandemic, one should rely on previous imaging and patient's history before making a misled diagnosis. Moreover, in patients who are known cases of cannonball pulmonary metastases and are being monitored for treatment response, new lesions, or lesions that show no decreased, 18F-FDG uptake in PET scan may not necessarily imply poor treatment response and simultaneous COVID-19 pneumonia is a possibility that needs to be ruled out (especially in suggestive clinical context) [19].

Author contribution Javid Azadbakht, Mahsa Masjedi Isfahani, Delbar Rastkhiz, and Hamidreza Talari were involved in the conception and design of the work. Javid Azadbakht, Mahsa Masjedi Isfahani, and Hamidreza Talari provided direction and guidance throughout the preparation of this manuscript. Mahsa Masjedi Isfahani and Delbar Rastkhiz collected data supporting this study. Javid Azadbakht wrote the manuscript. All authors approved the final version of the manuscript draft.

Data availability All data will be made available upon request from editor.

Code availability Not applicable.

\section{Declarations}

Ethics approval This study complies with guidelines for human studies. The affiliated institute's committee for research granted approval to this study.

Consent to participate A waiver of informed consent was granted for this retrospective study.

Consent for publication Both participants have consented to the submission of the case report to the journal.

Conflict of interest The authors declare that they have no conflict of interest.

\section{References}

1. COVID-19 Coronavirus pandemic 20212021 [updated August 25, 2021. Available from: https://www.worldometers.info/coron avirus/

2. World Health Organization. Statement on the second meeting of the International Health Regulations (2005) Emergency Committee regarding the outbreak of novel coronavirus (2019-nCoV). 2020

3. Bernheim A, Mei X, Huang M, Yang Y, Fayad ZA, Zhang N et al (2020) Chest CT findings in coronavirus disease-19 (COVID-19): relationship to duration of infection. Radiology 200463
4. Y Cao X Liu L Xiong K Cai 2020 Imaging and clinical features of patients with 2019 novel coronavirus SARS-CoV-2: a systematic review and meta-analysis J Med Virol 92914491459

5. M Chung A Bernheim X Mei N Zhang M Huang X Zeng 2020 CT imaging features of 2019 novel coronavirus (2019-nCoV) Radiology 2951202207

6. A Pakray D Walker A Figacz S Kilanowski C Rhodes S Doshi 2020 Imaging evaluation of COVID-19 in the emergency department Emerg Radiol 27579588

7. F Vernuccio D Giambelluca R Cannella FP Lombardo F Panzuto M Midiri 2020 Radiographic and chest CT imaging presentation and follow-up of COVID-19 pneumonia: a multicenter experience from an endemic area Emerg Radiol 276623632

8. Sheikh T, Tsai L, Hasan S, Tewari P, Assaly R (2020) Cannonball appearance of COVID-19 pneumonia: a novel presentation mimicking malignancy. J Med Case Rep Case Series 1(4)

9. MI Akerele P Wadhwa J Silva-Rodriguez W Hallett C Tsoumpas 2018 Validation of the physiological background correction method for the suppression of the spill-in effect near highly radioactive regions in positron emission tomography EJNMMI Phys 5 1121

10. Ammannagari N, Polu V (2013) 'Cannon ball'pulmonary metastases. Case Rep 2013:bcr2012008158

11. S Cull R Farhat A Krishna S Naydenov 2017 An uncommon presentation of pulmonary cannonball metastasis Chest 1524 A905

12. Falcone VA, Nardi I, Mesto M, Trotta V, Picca V (2019) An extremely rare case of adrenocortical cancer with cannon ball pulmonary metastasis. Monaldi Arch Chest Dis 89(3)

13. MA Shenoy P Gorukanti P Irukulla A Agarwal A Gajankush A Gour 2016 Cannon ball metastases: a rare pulmonary manifestation of diffuse large B-cell lymphoma Chest 1504 1107A

14. S Mittal A Chattopadhyay S Jain A Sharma 2019 Cannonball opacities in a pregnant woman Lancet Rheumatol $12 \mathrm{e} 132$

15. J Azadbakht D Khoramian ZS Lajevardi F Elikaii AH Aflatoonian B Farhood 2021 A review on chest CT scanning parameters implemented in COVID-19 patients: bringing low-dose CT protocols into play Egypt J Radiol Nucl Med 521110

16. KS Lee P Kullnig TE Hartman N Müller 1994 Cryptogenic organizing pneumonia: CT findings in 43 patients AJR Am J Roentgenol 1623543546

17. BP Pogatchnik KE Swenson H Sharifi H Bedi GJ Berry HH Guo 2020 Radiology-pathology correlation demonstrating organizing pneumonia in a patient who recovered from COVID-19 Am J Respir Crit Care Med 2024598599

18. T Ai Z Yang H Hou C Zhan C Chen W Lv 2020 Correlation of chest CT and RT-PCR testing for coronavirus disease 2019 (COVID-19) in China: a report of 1014 cases Radiology 2962 E32 E40

19. AJ Kavanal SR Jena R Kumar CK Das S Kumar BR Mittal 2021 Effect of COVID-19 on 18F-FDG PET/CT: is there a need to consider COVID-19 status before planning 18F-FDG PET/CT for oncologic evaluation? J Nucl Med Technol 493284285

Publisher's note Springer Nature remains neutral with regard to jurisdictional claims in published maps and institutional affiliations. 